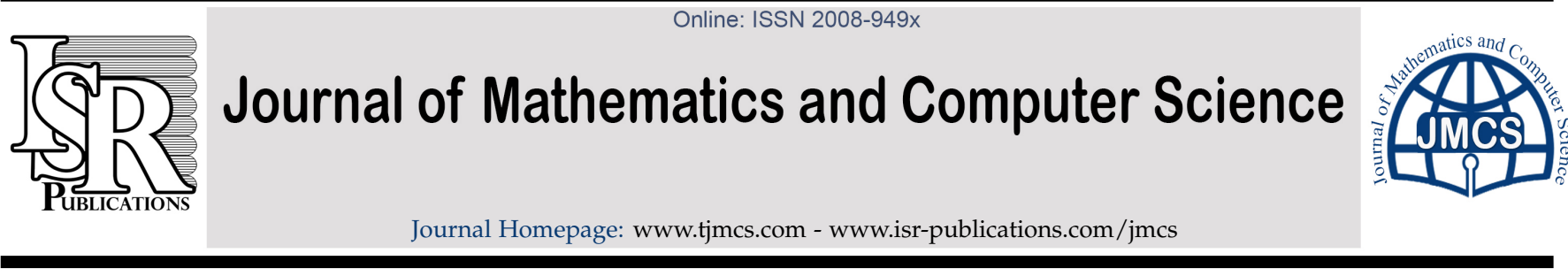

\title{
Solving fuzzy matrix games through a ranking value function method
}

\author{
Dong Qiu*, Yumei Xing, Shuqiao Chen \\ College of Science, Chongqing University of Post and Telecommunication, Chongqing, 400065, P. R. China.
}

\begin{abstract}
The objective of this paper is to establish the bi-matrix games with crisp payoffs based on ranking value function method. We obtain that the equilibrium solution of the game model can be translated into the optimal solution of the non-linear programming problem. Finally, to illustrate the effectiveness and correctness of the obtained model, an example is provided.
\end{abstract}

Keywords: Fuzzy bi-matrix game, equilibrium solution, non-linear programming problem.

2010 MSC: 15B15, 90C70.

(C)2018 All rights reserved.

\section{Introduction}

Generally speaking, the bi-matrix game model is accurate, when the bet is a small amount of money. In real-life, the relations between the interests of people are more complex. Particularly, in some areas of the economy, the interests of the two players is precisely the opposite. It is well known that these games are two person non-zero-sum game, which are called bi-matrix games for short. Therefore, much research in recent years has focus on bi-matrix game problems.

Mangasarian et al. [16] showed that the equilibrium solution of bi-matrix game is equivalent to the programming problem about linear constraints and a quadratic objective function. Hladik [10] introduced interval bi-matrix games and obtained the conclusion that the equilibrium solution of interval bi-matrix game can converted into the linear mixed integer programming problem. The methods of solving the interval bi-matrix games by a pair of auxiliary bilinear programming models is discussed by Fei and $\mathrm{Li}$ [9].

There have been a great number of studies in single objective fuzzy bi-matrix games $[2,3,11,12,15,19$, $24,25]$ and intuitionistic fuzzy bi-matrix games $[13,17,18]$. Maeda [15] discussed a bi-matrix game with fuzzy payoffs and proved the existence of Nash equilibrium in a fuzzy bi-matrix game. Vidyottama et al. [24] confirmed that a bi-matrix game with fuzzy goal and a crisp non-linear programming problem are of equal value. Vijay et al. [25] presented a bi-matrix game with fuzzy payoffs and fuzzy goals through a fuzzy relation method, and they showed that the solution is equal to two semi-infinite optimization

\footnotetext{
${ }^{*}$ Corresponding author

Email addresses: dongqiumath@163.com (Dong Qiu), x_ym2015@163com (Yumei Xing), cicanary@163.com (Shuqiao Chen)

doi: $10.22436 /$ jmcs.018.02.05
} 
problems. Li [12] proposed the concept of crisp parametric bi-matrix games and discussed the existence of equilibrium solutions of fuzzy bi-matrix games. Recently, Nayak and Pal [18] studied a bi-matrix game with intutionistic fuzzy goals. Subsequently, Li [13] discussed the way to deal with the bi-matrix games with payoffs of intuitionistic fuzzy sets via bilinear programming method and defuzzification ranking approach.

Although the research of bi-matrix game problems have been extensively studied in recent years, it needs to be further conducted from the perspective of the non-linear programming method. Gaining enlightenment from $[2,9,13]$, we will put forward a non-linear programming method for solving bimatrix games with payoffs matrices of ranking value function in this paper.

The outline of this paper is as follows. Section 2 describes the basic definitions and recalls the results that regard to crisp single-objective bi-matrix game. In Section 3, the bi-matrix games with crisp payoffs based on ranking value function method is established. And then the equivalence between the equilibrium solution of the game model problem and the optimal solution of a non-linear programming problem is interpreted. In Section 4, an example is provided to illustrate the effectiveness and correctness of the main results in this paper. Conclusions and suggestions for future research are given in the last section.

\section{Section title}

The following notations, definitions and results will be needed in the sequel.

We denote $\mathcal{K}_{\mathrm{C}}$ as the family of all bounded closed intervals in $\mathbb{R}[7]$, that is,

$$
\mathcal{K}_{C}=\left\{\left[a_{L}, a_{R}\right] \mid a_{L}, a_{R} \in \mathbb{R} \text { and } a_{L} \leqslant a_{R}\right\} .
$$

A fuzzy set $\widetilde{x}$ of $\mathbb{R}$ is characterized by a membership function $\mu_{\tilde{x}}: \mathbb{R} \rightarrow[0,1]$ [26]. For each such fuzzy set $\widetilde{x}$, we denote by $[\widetilde{x}]^{\alpha}=\left\{x \in \mathbb{R}: \mu_{\tilde{x}}(x) \geqslant \alpha\right\}$ for any $\alpha \in(0,1]$, its $\alpha$-level set. Define the set $[\widetilde{x}]^{0}$ by


non-empty bounded closed level sets $\left[\widetilde{x}^{\alpha}=\left[\widetilde{x}_{L}(\alpha), \widetilde{x}_{R}(\alpha)\right]\right.$ for all $\alpha \in[0,1]$, where $\left[\widetilde{x}_{L}(\alpha), \widetilde{x}_{R}(\alpha)\right]$ denotes a closed interval with the left end point $\widetilde{x}_{L}(\alpha)$ and the right end point $\widetilde{x}_{R}(\alpha)$ [8]. We denote the class of fuzzy numbers by $\mathscr{F}$.

Definition 2.1 ([4]). Let $\tau: \mathscr{F} \rightarrow \mathbb{R}$ be a function defined by

$$
\tau(\widetilde{x})=\int_{0}^{1} \alpha\left[\widetilde{x}_{L}(\alpha)+\widetilde{x}_{R}(\alpha)\right] d \alpha
$$

for all $\tilde{x} \in \mathscr{F}$. Then $\tau$ is called ranking value function.

Definition 2.2 ([4]). Suppose that $\widetilde{x}$ and $\widetilde{y}$ are two fuzzy numbers. Then $\widetilde{x}$ precedes $\widetilde{y}(\widetilde{x} \preceq \widetilde{y})$ if

$$
\int_{0}^{1} \alpha\left[\widetilde{x}_{L}(\alpha)+\widetilde{x}_{R}(\alpha)\right] d \alpha \leqslant \int_{0}^{1} \alpha\left[\widetilde{y}_{L}(\alpha)+\widetilde{y}_{R}(\alpha)\right] d \alpha .
$$

Note that the order relation $\preceq$ is reflexive and transitive. Moreover, any two elements of $\mathscr{F}$ are comparable under the ordering $\preceq$. For more details see [22, 23, 27].

Lemma 2.3 ([4]). For $\widetilde{x}, \widetilde{y} \in \mathscr{F}$, then $\widetilde{x} \preceq \widetilde{y}$ if and only if $\tau(\widetilde{x}) \leqslant \tau(\widetilde{y})$.

Definition 2.4 ([14]). Let $\widetilde{a}_{i}$ be fuzzy numbers and $x_{i} \geqslant 0(i=1,2, \ldots, n)$ be an real numbers. Then, $\sum_{i=1}^{n} \widetilde{a}_{i} x_{i}$ is a fuzzy number.

In the following, we shall describe a crisp bi-matrix game model in [1, 2].

Definition 2.5 ([2]). The set of mixed strategies for player I is denoted by

$$
S^{m}=\left\{x=\left(x_{1}, x_{2}, \cdots, x_{m}\right)^{\top} \in \mathbb{R}^{m} \mid \sum_{i=1}^{m} x_{i}=1, x_{i} \geqslant 0, i=1,2, \cdots, m\right\} .
$$


Similarly, The set of mixed strategies for player II is denoted by

$$
S^{n}=\left\{y=\left(y_{1}, y_{2}, \cdots, y_{n}\right)^{\top} \in \mathbb{R}^{n} \mid \sum_{j=1}^{n} y_{j}=1, y_{j} \geqslant 0, j=1,2, \cdots, n\right\},
$$

where $x^{\top}$ is the transposition of $x$, and $\mathbb{R}^{m}$ and $\mathbb{R}^{n}$ are $m$ - and $n$-dimensional Euclidean spaces, respectively.

A payoff matrix of the Player I and Player II in bi-matrix game are denoted by $([1,2])$

$$
A=\left(\begin{array}{ccc}
a_{11} & \cdots & a_{1 n} \\
\vdots & \ddots & \vdots \\
a_{m 1} & \cdots & a_{m n}
\end{array}\right) \quad \text { and } B=\left(\begin{array}{ccc}
b_{11} & \cdots & b_{1 n} \\
\vdots & \ddots & \vdots \\
b_{m 1} & \cdots & b_{m n}
\end{array}\right)
$$

respectively. Assume that the player I and player II both are maximized players. A bi-matrix game (BG) model in [2] is taken as

$$
B G=\left(S^{m}, S^{n}, A, B\right) .
$$

Definition $2.6([1,2])$. Let $A$ be a payoff matrix of the Player I. When Player I and Player II choose mixed strategies $x \in S^{m}$ and $y \in S^{n}$, respectively, an expected payoff of Player I is denoted by

$$
E(x, y, A)=x^{\top} A y=\sum_{i=1}^{m} \sum_{j=1}^{n} a_{i j} x_{i} y_{j}
$$

Similarly, let B be a payoff matrix of the Player II, an expected payoff of Player II is denoted by

$$
E(x, y, B)=x^{\top} B y=\sum_{i=1}^{m} \sum_{j=1}^{n} b_{i j} x_{i} y_{j} .
$$

Definition $2.7([1,2]) \cdot(\bar{x}, \bar{y}) \in S^{m} \times S^{n}$ is called the pair of equilibrium solution of the game (BG) model if

$$
x^{\top} A \bar{y} \leqslant \bar{x}^{\top} A \bar{y}, \forall x \in S^{m}, \quad \bar{x}^{\top} B y \leqslant \bar{x}^{\top} B \bar{y}, \forall y \in S^{n} .
$$

Theorem 2.8 ([1, 2]). For any a given game (BG) model, $(\bar{x}, \bar{y})$ is an equilibrium solution of game (BG) model if and only if it is a optimal solution the following problem (NLP1) model,

$$
\begin{array}{cl}
\max & \sigma_{1}+\sigma_{2}-p-q \\
\text { subject to } \quad & A_{i} y \leqslant p,(i=1,2, \ldots m), \\
& B_{j}^{\top} x \leqslant q,(j=1,2, \ldots n), \\
& x^{\top} A y \geqslant \sigma_{1}, \\
& x^{\top} B y \geqslant \sigma_{2}, \\
& 0 \leqslant \lambda \leqslant 1, \\
x & \in S^{m}, y \in S^{n} .
\end{array}
$$

\section{A bi-matrix game model based on ranking value function}

In this section, we will establish a bi-matrix game model through a ranking value function. Let $S^{m}$ and $S^{n}$ be introduced in Section 2. Suppose that the elements of $\widetilde{A}$ and $\widetilde{B}$ be fuzzy numbers. Therefore, the bi-matrix game with fuzzy payoffs [2], denoted by BGFP, can be presented as

$$
B G F P=\left(S^{m}, S^{n}, \widetilde{A}, \widetilde{B}\right) .
$$


Definition 3.1. Let $\widetilde{A}$ be fuzzy payoff matrix of the Player I. When Player I and Player II choose mixed strategies $x \in S^{m}$ and $y \in S^{n}$, respectively, a fuzzy expected payoff of Player I is denoted by

$$
E(x, y, \widetilde{A})=x^{\top} \widetilde{A} y=\sum_{i=1}^{m} \sum_{j=1}^{n} \widetilde{a}_{i j} x_{i} y_{j} .
$$

Similarly, let $\widetilde{B}$ be fuzzy payoff matrix of the Player II, a fuzzy expected payoff of Player II is denoted by

$$
E(x, y, \widetilde{B})=x^{\top} \widetilde{B} y=\sum_{i=1}^{m} \sum_{j=1}^{n} \widetilde{b}_{i j} x_{i} y_{j}
$$

Definition 3.2. Let $(x, y) \in S^{m} \times S^{n}$. If $x \in S^{m}$ and $y \in S^{n}$ satisfy the following conditions,

$$
x^{\top} \widetilde{A} \bar{y} \preceq \bar{x}^{\top} \widetilde{A} \bar{y}, \forall x \in S^{m}, \quad \bar{x}^{\top} \widetilde{B} y \preceq \bar{x}^{\top} \widetilde{B} \bar{y}, \forall y \in S^{n},
$$

then $(\bar{x}, \bar{y}) \in S^{m} \times S^{n}$ is called the equilibrium solution of (BGFP).

In order to get the equilibrium solution of (BGFP), by Definition 2.1, we introduce payoff matrices based on ranking value function of Player I and Player II as follows:

$$
\tau(\widetilde{A})=\left(\begin{array}{ccc}
\tau\left(\widetilde{a}_{11}\right) & \cdots & \tau\left(\widetilde{a}_{1 n}\right) \\
\vdots & \ddots & \vdots \\
\tau\left(\widetilde{a}_{m 1}\right) & \cdots & \tau\left(\widetilde{a}_{m n}\right)
\end{array}\right) \quad \text { and } \quad \tau(\widetilde{B})=\left(\begin{array}{cccc}
\tau\left(\widetilde{b}_{11}\right) & \cdots & \tau\left(\widetilde{b}_{1 n}\right) \\
\vdots & \ddots & \vdots \\
\tau\left(\widetilde{b}_{m 1}\right) & \cdots & \tau\left(\widetilde{b}_{m n}\right)
\end{array}\right)
$$

where

$$
\tau\left(\widetilde{a}_{i j}\right)=\int_{0}^{1} \alpha\left[\left(\widetilde{a}_{i j}\right)_{L}(\alpha)+\left(\widetilde{a}_{i j}\right)_{R}(\alpha)\right] d \alpha \quad \text { and } \quad \tau\left(\widetilde{b}_{i j}\right)=\int_{0}^{1} \alpha\left[\left(\widetilde{b}_{i j}\right)_{L}(\alpha)+\left(\widetilde{b}_{i j}\right)_{R}(\alpha)\right] d \alpha
$$

Thus, we obtain that the bi-matrix game based on ranking value function, denoted by BGRVFP, can be presented as

$$
\text { BGRVFP }=\left(S^{m}, S^{n}, \tau(\widetilde{A}), \tau(\widetilde{B})\right) .
$$

Next, we give an equivalent definition of equilibrium solution of (BGFP).

Definition 3.3. Let $\alpha \in[0,1],(x, y) \in S^{m} \times S^{n}$. If $x \in S^{m}$ and $y \in S^{n}$ satisfy following the conditions:

$$
x^{\top} \tau(\widetilde{A}) \bar{y} \leqslant \bar{x}^{\top} \tau(\widetilde{A}) \bar{y}, \forall x \in S^{m}, \quad \bar{x}^{\top} \tau(\widetilde{B}) y \leqslant \bar{x}^{\top} \tau(\widetilde{B}) \bar{y}, \forall y \in S^{n},
$$

then, $(\bar{x}, \bar{y}) \in S^{m} \times S^{n}$ is called the equilibrium solution of (BGRVFP).

Theorem 3.4. The point $(\bar{x}, \bar{y}) \in S^{m} \times S^{n}$ is the equilibrium solution of (BGFP) if and only if it is the equilibrium solution of (BGRVFP).

Proof. Since the elements of $\widetilde{A}$ and $\widetilde{B}$ be fuzzy numbers, by Definition 2.4, we obtain that the elements of $x^{\top} \widetilde{A} \bar{y}, \tilde{x}^{\top} \widetilde{A} \bar{y}, \bar{x}^{\top} \widetilde{B} y$, and $\bar{x}^{\top} \widetilde{B} \bar{y}$ are fuzzy numbers. Using Lemma 2.3, we have

$$
x^{\top} \widetilde{A} \bar{y} \preceq \bar{x}^{\top} \widetilde{A} \bar{y} \Leftrightarrow x^{\top} \tau(\widetilde{A}) \bar{y} \leqslant \bar{x}^{\top} \tau(\widetilde{A}) \bar{y}, \forall x \in S^{m}, \quad \bar{x}^{\top} \widetilde{B} y \preceq \bar{x}^{\top} \widetilde{B} \bar{y} \Leftrightarrow \bar{x}^{\top} \tau(\widetilde{B}) y \leqslant \bar{x}^{\top} \tau(\widetilde{B}) \bar{y}, \forall y \in S^{n} .
$$

Thus, The point $(\bar{x}, \bar{y}) \in S^{m} \times S^{n}$ is the equilibrium solution of (BGFP) if and only if it is the equilibrium solution of (BGRVFP).

Therefore, we convert the equilibrium solution of (BGFP) to the equilibrium solution of (BGRVFP). Now, we consider the game (BGRVFP) model. By Definition 3.3, we conclude the following theorem. 
Theorem 3.5. $(\bar{x}, \bar{y}) \in S^{m} \times S^{n}$ is the equilibrium solution of (BGRVFP) if only and if it is the optimal solution of the following problem (P1)

$$
\max _{x} x^{\top} \tau(\widetilde{A}) \bar{y}+\max _{y} \bar{x}^{\top} \tau(\widetilde{B}) y \quad \text { such that } \forall x \in S^{m}, \forall y \in S^{n} .
$$

Proof. By Definition 3.3, we can obtain that the equilibrium solution of (BGRVFP) is equal to the following two problems (P2) and (P3).

$$
\begin{array}{ll}
\max _{x} x^{\top} \tau(\widetilde{A}) \bar{y} & \text { such that } \forall x \in S^{m}, \\
\max _{y} \bar{x}^{\top} \tau(\widetilde{B}) y & \text { such that } \forall y \in S^{n} .
\end{array}
$$

In terms of $\forall x \in S^{m}$ and $\forall y \in S^{n}$, the constraints of (P2) and (P3) are separable. Then, the problems (P2) and (P3) can be rewritten as (P1). Thus, the proof of this theorem is completed.

Based on the above discusses, we can get the following theorem.

Theorem 3.6. For any a given game (BGRVFP) model, $(\bar{x}, \bar{y})$ is an equilibrium solution of (BGRVFP) if and only if it is an optimal solution of the following problem (NLP2),

$$
\begin{gathered}
\max \sigma_{1}+\sigma_{2}-p-q \\
\text { subject to } \quad \tau\left(\widetilde{A}_{i}\right) y \leqslant p,(i=1,2, \ldots m), \\
\tau\left(\widetilde{B}_{j}^{\top}\right) x \leqslant q,(j=1,2, \ldots n), \\
x^{\top} \tau(\widetilde{A}) y \geqslant \sigma_{1}, \\
x^{\top} \tau(\widetilde{B}) y \geqslant \sigma_{2}, \\
0 \leqslant \lambda \leqslant 1, \\
x \in S^{m}, y \in S^{n} .
\end{gathered}
$$

Proof. From (2.1), (2.2), and conditions of (NLP2), we can directly get

$$
\begin{aligned}
\sigma_{1}+\sigma_{2}-p-q & \leqslant x^{\top} \tau(\widetilde{A}) y+x^{\top} \tau(\widetilde{B}) y-\sum_{i=1}^{m} x_{i} p-\sum_{j=1}^{n} y_{j} q \\
& =x^{\top} \tau(\widetilde{A}) y+x^{\top} \tau(\widetilde{B}) y-\sum_{i=1}^{m} x_{i} \tau\left(\widetilde{A}_{i}\right) y-\sum_{j=1}^{n} y_{j} \tau\left(\widetilde{B}_{j}^{\top}\right) x \\
& =x^{\top} \tau(\widetilde{A}) y+x^{\top} \tau(\widetilde{B}) y-x^{\top} \tau(\widetilde{A}) y-x^{\top} \tau(\widetilde{B}) y=0 .
\end{aligned}
$$

Therefore, observe that

$$
\max \sigma_{1}+\sigma_{2}-p-q \leqslant 0 .
$$

Further, we get that $\left(\bar{x}, \bar{y}, \bar{p}, \bar{q}, \bar{\sigma}_{1}, \bar{\sigma}_{2}\right)$ is an optimal solution of (NLP2). Here $\bar{p}=\bar{\sigma}_{1}=\bar{x}^{\top} \tau(\widetilde{A}) \bar{y}$, $\bar{q}=\bar{\sigma}_{2}=\bar{x}^{\top} \tau(\widetilde{B}) \bar{y}$. Hence, we get that maximum value of objective function of (NLP2) is 0 .

Sufficiency. Suppose that $\left(\bar{x}, \bar{y}, \bar{p}, \bar{q}, \bar{\sigma}_{1}, \bar{\sigma}_{2}\right)$ be the optimal solution of problem (NLP2). Based on the above discussion, we have

$$
\max \bar{\sigma}_{1}+\bar{\sigma}_{2}-\bar{p}-\bar{q}=0 .
$$

Combining with (2.1), (2.2), and conditions of (NLP2), we obtain

$$
x^{\top} \tau(\tilde{A}) \bar{y}=\sum_{i=1}^{m} x_{i} \tau\left(\widetilde{A}_{i}\right) \bar{y} \leqslant \sum_{i=1}^{m} x_{i} \bar{p}=\bar{p},
$$




$$
\begin{aligned}
& \bar{x}^{\top} \tau(\widetilde{B}) y=\sum_{j=1}^{n} y_{j} \tau\left(\widetilde{B}_{j}^{\top}\right) \bar{x} \leqslant \sum_{j=1}^{n} y_{j} \bar{q}=\bar{q}, \\
& \bar{x}^{\top} \tau(\widetilde{A}) \bar{y} \geqslant \bar{\sigma}_{1}, \\
& \bar{x}^{\top} \tau(\widetilde{B}) \bar{y} \geqslant \bar{\sigma}_{2} .
\end{aligned}
$$

From (3.3), (3.6), and (3.7), we have

$$
\bar{p}+\bar{q}=\bar{\sigma}_{1}+\bar{\sigma}_{2} \leqslant \bar{x}^{\top} \tau(\widetilde{A}) \bar{y}+\bar{x}^{\top} \tau(\widetilde{B}) \bar{y} .
$$

That is,

$$
\bar{p}+\bar{q}-\bar{x}^{\top} \tau(\widetilde{B}) \bar{y} \leqslant \bar{x}^{\top} \tau(\widetilde{A}) \bar{y} .
$$

In condition (3.4), let $x=\bar{x}$, we have

$$
\bar{p}+\bar{q}-\bar{x}^{\top} \tau(\widetilde{B}) \bar{y} \leqslant \bar{x}^{\top} \tau(\widetilde{A}) \bar{y} \leqslant \bar{p} .
$$

Hence,

$$
\bar{x}^{\top} \tau(\widetilde{B}) \bar{y} \geqslant \bar{q}
$$

In condition (3.5), let $y=\bar{y}$, we have

$$
\bar{p}+\bar{q}-\bar{x}^{\top} \tau(\widetilde{A}) \bar{y} \leqslant \bar{x}^{\top} \tau(\widetilde{B}) \bar{y} \leqslant \bar{q} .
$$

Thus,

$$
\bar{x}^{\top} \tau(\widetilde{A}) \bar{y} \geqslant \bar{p} .
$$

Combining with (3.4), (3.5), (3.8), and (3.9), we obtain

$$
x^{\top} \tau(\widetilde{A}) \bar{y} \leqslant \bar{x}^{\top} \tau(\widetilde{A}) \bar{y}, \forall x \in S^{m}, \quad \bar{x}^{\top} \tau(\widetilde{B}) y \leqslant \bar{x}^{\top} \tau(\widetilde{B}) \bar{y}, \forall y \in S^{n} .
$$

By Definition 3.3, we get that $(\bar{x}, \bar{y})$ is an equilibrium solution of (BGRVFP).

Necessity. Let $(\bar{x}, \bar{y})$ be an equilibrium solution of (BGRVFP). Utilizing Definition 3.3, we can get

$$
x^{\top} \tau(\widetilde{A}) \bar{y} \leqslant \bar{x}^{\top} \tau(\widetilde{A}) \bar{y}, \quad \forall x \in S^{m}, \quad \bar{x}^{\top} \tau(\widetilde{B}) y \leqslant \bar{x}^{\top} \tau(\widetilde{B}) \bar{y}, \quad \forall y \in S^{n} .
$$

Since $\bar{p}=\bar{\sigma}_{1}=\bar{x}^{\top} \tau(\widetilde{A}) \bar{y}, \bar{q}=\bar{\sigma}_{2}=\bar{x}^{\top} \tau(\widetilde{B}) \bar{y}$. Then,

$$
\bar{\sigma}_{1}+\bar{\sigma}_{2}-\bar{p}-\bar{q}=0 .
$$

However, from (3.2), we get

$$
0=\bar{\sigma}_{1}+\bar{\sigma}_{2}-\bar{p}-\bar{q}=\max \sigma_{1}+\sigma_{2}-p-q .
$$

Thus, $(\bar{x}, \bar{y})$ is an optimal solution the problem (NLP2).

Corollary 3.7. The optimal solution of the following problems (P1) is equal to the optimal solution the problem (NLP2).

Corollary 3.8. If $\tau(\widetilde{A})=-\tau(\widetilde{B})$, the problem (NLP2) can become the following primal-dual pair of problems (LP1) and (LD1).

$$
\begin{array}{r}
\max -p \text { such that } \tau\left(\widetilde{A}_{i}\right) y \leqslant p,(i=1,2, \ldots, m), \quad \forall y \in S^{n}, \\
\max -q \text { such that }-\tau\left(\widetilde{A}_{j}^{\top}\right) x \leqslant q,(j=1,2, \ldots, n), \quad \forall x \in S^{m} .
\end{array}
$$

Now, let $-\mathrm{q}=\lambda$, then problems (LP1) and (LD1) can rewritten as

$$
\begin{aligned}
\min p & \text { such that } \tau\left(\widetilde{A}_{i}\right) y \leqslant p,(i=1,2, \ldots, m), \quad \forall y \in S^{n}, \\
\max \lambda & \text { such that } \tau\left(\widetilde{A}_{j}^{\top}\right) x \geqslant \lambda,(j=1,2, \ldots, n), \quad \forall x \in S^{m} .
\end{aligned}
$$


Theorem 3.9. For any a given game (BGRVFP) model, if the elements of $\widetilde{\mathrm{A}}$ and $\widetilde{\mathrm{B}}$ are triangular fuzzy numbers, then $(\bar{x}, \bar{y})$ is an equilibrium solution of (BGRVFP) if and only if it is a optimal solution of the problem (NLP3).

$$
\begin{gathered}
\max \sigma_{1}+\sigma_{2}-p-q \\
\text { subject to } \sum_{j=1}^{n} \frac{a_{i j}+4 a_{i j}+\grave{a}_{i j}}{6} y_{j} \leqslant p,(i=1,2, \ldots, m), \\
\sum_{i=1}^{m} \frac{\hat{b}_{i j}+4 b_{i j}+\grave{b}_{i j}}{6} x_{i} \leqslant q,(j=1,2, \ldots, n), \\
\sum_{i=1}^{m} \sum_{j=1}^{n} \frac{\hat{a}_{i j}+4 a_{i j}+\grave{a}_{i j}}{6} x_{i} y_{j} \geqslant \sigma_{1}, \\
\sum_{i=1}^{m} \sum_{j=1}^{n} \frac{\hat{b}_{i j}+4 b_{i j}+\grave{b}_{i j}}{6} x_{i} y_{j} \geqslant \sigma_{2}, \\
x \in S^{m}, y \in S^{n} .
\end{gathered}
$$

Proof. Since the elements of $\widetilde{A}$ and $\widetilde{B}$ are triangular fuzzy numbers,

$$
\left[\widetilde{\mathrm{a}}_{i j}\right]^{\alpha}=\left[\left(\widetilde{\mathrm{a}}_{i j}\right)_{\mathrm{L}}(\alpha),\left(\widetilde{\mathrm{a}}_{i j}\right)_{R}(\alpha)\right]=\left[\mathfrak{a}_{i j}+\alpha\left(\mathrm{a}_{i j}-\mathfrak{a}_{i j}\right), \grave{a}_{i j}-\alpha\left(\grave{a}_{i j}-a_{i j}\right)\right]
$$

and

$$
\left[\tilde{b}_{i j}\right]^{\alpha}=\left[\left(\tilde{b}_{i j}\right)_{L}(\alpha),\left(\tilde{b}_{i j}\right)_{R}(\alpha)\right]=\left[\hat{b}_{i j}+\alpha\left(b_{i j}-\hat{b}_{i j}\right), \grave{b}_{i j}-\alpha\left(\grave{b}_{i j}-b_{i j}\right)\right]
$$

for $i=1,2, \ldots, m ; j=1,2, \ldots, n$. By Definition 2.1, we can get

$$
\begin{aligned}
& \tau\left(\widetilde{a}_{i j}\right)=\int_{0}^{1} \alpha\left[\left(\widetilde{a}_{i j}\right)_{L}(\alpha)+\left(\widetilde{a}_{i j}\right)_{R}(\alpha)\right] d \alpha=\int_{0}^{1} \alpha\left[\hat{a}_{i j}+\alpha\left(a_{i j}-\dot{a}_{i j}\right)+\grave{a}_{i j}-\alpha\left(\grave{a}_{i j}-a_{i j}\right)\right] d \alpha=\frac{\dot{a}_{i j}+4 a+\grave{a}_{i j}}{6}, \\
& \tau\left(\widetilde{b}_{i j}\right)=\int_{0}^{1} \alpha\left[\left(\widetilde{b}_{i j}\right)_{L}(\alpha)+\left(\widetilde{b}_{i j}\right)_{R}(\alpha)\right] d \alpha=\int_{0}^{1} \alpha\left[\hat{b}_{i j}+\alpha\left(b_{i j}-\grave{b}_{i j}\right)+\grave{b}_{i j}-\alpha\left(\grave{b}_{i j}-b_{i j}\right)\right] d \alpha=\frac{\dot{b}_{i j}+4 a+\grave{b}_{i j}}{6} .
\end{aligned}
$$

By using Theorem 3.6, we have that $(\bar{x}, \bar{y})$ is an equilibrium solution of (BGRVFP) if and only if it is a optimal solution of the problem (NLP3).

\section{Example}

In order to illustrate the effectiveness and correctness of the obtained model, we now consider the following fuzzy bi-matrix game (BGRVFP) model.

Example 4.1. For any a given game (BGRVFP) model, the fuzzy payoff matrices of the Player I and Player II are taken as

$$
\widetilde{A}=\left(\begin{array}{lll}
(1.80,2.00,2.20) & (2.90,3.00,3.10) & (7.75,8.00,8.20) \\
(7.75,8.00,8.10) & (3.50,4.00,4.20) & (0.90,1.00,1.20)
\end{array}\right)
$$

and

$$
\widetilde{\mathrm{B}}=\left(\begin{array}{lll}
(4.80,5.00,5.50) & (1.80,2.00,2.75) & (4.80,5.00,5.50) \\
(5.75,6.00,6.40) & (0.75,1.00,1.20) & (3.50,4.00,4.50)
\end{array}\right)
$$

respectively.

We now solve this problem with the above model. By using (3.1), we get the following payoff matrices of Player I and Player II ,

$$
\tau(\widetilde{A})=\left(\begin{array}{ccc}
2.00 & 3.00 & 7.99 \\
7.975 & 3.95 & 1.02
\end{array}\right) \text { and } \tau(\widetilde{B})=\left(\begin{array}{ccc}
5.05 & 2.09 & 5.05 \\
6.025 & 0.99 & 4.00
\end{array}\right),
$$

respectively. Thus now utilizing conditions of (NLP3), we obtain the following problem (NLP4). 


$$
\begin{aligned}
\max & \sigma_{1}+\sigma_{2}-p-q \\
\text { subject to } & 2.00 y_{1}+3.00 y_{2}+7.99 y_{3} \leqslant p, \\
& 7.975 y_{1}+3.95 y_{2}+1.02 y_{3} \leqslant p, \\
& 5.05 x_{1}+6.025 x_{2} \leqslant q, \\
& 2.09 x_{1}+0.99 x_{2} \leqslant q, \\
& 5.05 x_{1}+4.00 x_{2} \leqslant q, \\
& 2.00 x_{1} y_{1}+7.975 x_{2} y_{1}+3.00 x_{1} y_{2}+3.95 x_{2} y_{2}+7.99 x_{1} y_{3}+1.02 x_{2} y_{3} \geqslant \sigma_{1}, \\
& 5.05 x_{1} y_{1}+6.025 x_{2} y_{1}+2.09 x_{1} y_{2}+0.99 x_{2} y_{2}+5.05 x_{1} y_{3}+4.00 x_{2} y_{3} \geqslant \sigma_{2}, \\
& x \in S^{m}, y \in S^{n} .
\end{aligned}
$$

We obtain that the optimal solution of the problem (NLP4) is $\left(\left(\bar{x}_{1}=0.4, \bar{x}_{2}=0.6\right),\left(\bar{y}_{1}=0.4, \bar{y}_{2}=\right.\right.$ $\left.\left.0.3, \bar{y}_{3}=0.3\right)\right)$. By Theorem 3.9, we can get that $\left(\left(\bar{x}_{1}=0.4, \bar{x}_{2}=0.6\right),\left(\bar{y}_{1}=0.4, \bar{y}_{2}=0.3, \bar{y}_{3}=0.3\right)\right)$ is an equilibrium solution of the above game (BGRVFP) model.

\section{Conclusion}

In this paper, we have presented the bi-matrix game model with crisp payoffs based on ranking value function method. It is shown that the equilibrium solution of the game model can be translated into the optimal solution of a non-linear programming problem. In recent years, more and more researchers have studied the bi-matrix game model under the environment of intuitionistic fuzzy and entropy [5, 6, 13, 17, $18,20,21]$. We believe that our research is cost-effective and it offers an understanding of the bi-matrix game model. Further studies involving the game model of intuitionistic fuzzy (entropy) environment are expected to be discussed.

\section{Acknowledgment}

This work was supported by The National Natural Science Foundations of China (Grant no. 11671001 and 61472056).

\section{References}

[1] T. Başar, G. J. Olsder, Dynamic Noncooperative Game Theory, Academic press, London, (1995). 2, 2, 2.6, 2.7, 2.8

[2] C. R. Bector, S. Chandra, Fuzzy mathematical programming and fuzzy matrix games, Springer, Germany, (2005). 1, 2, 2.5, 2, 2.6, 2.7, 2.8, 3

[3] D. Blackwell, An analog of the minimax theorem for vector payoff, Pacific J. Math., 6 (1956), 1-8. 1

[4] Y. Chalco-Canoa, A. Rufián-Lizana, H. Román-Flores, R. Osuna-Gómez, A note on generalized convexity for fuzzy mappings through a linear ordering, Fuzzy Sets and Systems, 231 (2013), 70-83. 2.1, 2.2, 2.3

[5] C. B. Das, S. K. Roy, Fuzzy based GA to multi-objective entropy bimatrix game, Opsearch, 50 (2013), 125-140. 5

[6] A. DeLuca, S. Termini, A definition of a nonprobabilistic entropy in the setting of fuzzy sets theory, Information and Control, 20 (1972), 301-312. 5

[7] P. Diamond, P. Kloeden, Metric spaces of fuzzy sets, Fuzzy Sets and Systems, 35 (1990), 241-249. 2

[8] D. J. Dubois, H. Prade, Fuzzy sets and systems-theory and application, Academic Press, New York, (1980). 2

[9] W. Fei, D.-F. Li, Bilinear Programming Approach to Solve Interval Bimatrix Games in Tourism Planning Management, Int. J. Fuzzy Syst., 18 (2016), 504-510. 1

[10] M. Hladík, Interval valued bimatrix games, Kybernetika, 46 (2010), 435-446. 1

[11] M. Larbani, Solving bi-matrix games with fuzzy payoffs by introducing nature as a third player, Fuzzy Sets and Systems, 160 (2009), 657-666. 1

[12] C.-L. Li, Characterization of the Equilibrium Strategy of Fuzzy Bimatrix Games Based on Fuzzy Variables, J. Appl. Math., 2012 (2012), 15 pages. 1

[13] D.-F. Li, Decision and Game Theory in Management with Intuitionistic Fuzzy Sets, Springer-verlag, Heidelberg, (2014). 1,5

[14] L. Li, S. Liu, J. Zhang, On fuzzy generalized convex mappings and optimality conditions for fuzzy weakly univex mappings, Fuzzy Sets and Systems, 280 (2015), 107-132. 2.4 
[15] T. Maeda, Characterization of the equilibrium strategy of the bimatrix game with fuzzy payoff, J. Math. Anal. Appl., 251 (2000), 885-896. 1

[16] O. L. Mangasarian, H. Stone, Two person non zero sum game and quadratic programming, J. Math. Anal. Appl., 9 (1964), 348-355. 1

[17] J.-X. Nan, M.-J. Zhang, D.-F. Li, Intuitionistic fuzzy programming models for matrix games with payoffs of trapezoidal intuitionistic fuzzy numbers, Int. J. Fuzzy Syst., 16 (2014), 444-456. 1, 5

[18] P. K. Nayak, M. Pal, Bimatrix games with intiutionstic fuzzy goals, Iran. J. Fuzzy Syst., 7 (2010), 65-79. 1, 5

[19] M. Panigrahi, G. Panda, S. Nanda, Convex fuzzy mapping with differentiability and its application in fuzzy optimization, European J. Oper. Res., 185 (2008), 47-62. 1

[20] S. K. Roy, Fuzzy programming approach to two-person multicriteria bimatrix games, J. Fuzzy Math., 15 (2007), $141-153$. 5

[21] S. K. Roy, M. P. Biswal, R. N. Tiwari, An approach to multi-objective bimatrix games for Nash equilibrium solutions, Ric. Oper., 30 (2001), 56-63. 5

[22] Y. R. Syau, E. Stanley Lee, Fuzzy Weirstrass theorem and convex fuzzy mappings, Comput. Math. Appl., 51 (2006), 1741-1750. 2

[23] Y. R. Syau, E. Stanley Lee, Preinvexity and F1-convexity of fuzzy mappings through a linear ordering, Comput. Math. Appl., 51 (2006), 405-418. 2

[24] V. Vidyottama, S. Chandra, C. R. Bector, Bi-matrix game with fuzzy goals and fuzzy payoffs, Fuzzy Optim. Decis. Mak., 3 (2004), 327-344. 1

[25] V. Vijay, A. Mehra, S. Chandra, C. R. Bector, Fuzzy matrix games via a fuzzy relation approach, Fuzzy Optim. Decis. Mak., 6 (2007), 299-314. 1

[26] L. A. Zadeh, Fuzzy sets, Information and Control, 8 (1965), 338-353. 2

[27] H.-J. Zimmermann, Fuzzy programming and linear programming with several objective functions, Fuzzy Sets and Systems, 1 (1978), 45-55. 2 